\title{
Normal Mode Analysis of the High Speed Channel Flow in a Bidisperse Porous Medium
}

\author{
Eugen Magyari
}

Received: 9 January 2013 / Accepted: 19 January 2013 / Published online: 7 February 2013

C Springer Science+Business Media Dordrecht 2013

\begin{abstract}
The linear Darcy-Brinkman model of the high speed flow in a bidisperse porous medium proposed by Nield and Kuznetsov (Transport Phenomena in Porous Media, 2005) is revisited in this paper. For the steady unidirectional flow in a parallel plane channel the exact analytical solutions for the fluid velocities are worked out by the normal-mode reduction of the governing equations. The limiting cases of the weak and strong momentum transfer between the flows in the fracture and porous phases are discussed in detail. A comparison to the nonlinear Forchheimer extension of the model proposed recently by Nield and Kuznetsov (Transport Porous Media, 2013) shows that, in the considered parameter range, the nonlinear effect of the Forchheimer drag is negligibly small. Even the simplest zero-momentum transfer solution yields an acceptable approximation.
\end{abstract}

Keywords Bidisperse medium · Forced convection · High speed flow · Parallel plane channel $\cdot$ Normal modes $\cdot$ Forchheimer drag

\section{Introduction and Problem Formulation}

In a recent paper of Nield and Kuznetsov (2013) the steady unidirectional forced convection high speed flow in a parallel plane channel filled with a bidisperse porous medium has been investigated assuming a nonlinear Forchheimer-extension of their linear Darcy-Brinkman model proposed few years ago (Nield and Kuznetsov 2005; Nield and Bejan 2006, p. 26). For some representative values of the involved parameters, the fluid velocities in the fracture phase and the porous phase of the bidisperse medium have been calculated numerically. The present paper revisits this forced convection problem, its aim being threefold: (i) to give the exact analytical solution of the linear Darcy-Brinkman model by a normal-mode reduction of the governing coupled differential equations, (ii) to elaborate the limiting cases of the

E. Magyari $(\varangle)$

Departement Physik, Theoretische Physik, Universität Basel,

Klingelbergstr. 82, 4056 Basel, Switzerland

e-mail: magyari@bluewin.ch 
weak and strong momentum transfer between the flows in the fracture and porous phases in detail and (iii) to evaluate the effect of the Forchheimer drag by a comparison of the exact results to the novel findings of Nield and Kuznetsov (2013).

The governing dimensionless equations of the Darcy-Brinkman-Forchheimer model of the unidirectional channel flow considered by Nield and Kuznetsov (2013) are

$$
\begin{aligned}
& \frac{\mathrm{d}^{2} u_{\mathrm{f}}}{\mathrm{d} y^{2}}=\frac{\gamma_{\mathrm{f}}}{\sqrt{D a_{\mathrm{f}}}} u_{\mathrm{f}}^{2}+\frac{u_{\mathrm{f}}}{D a_{\mathrm{f}}}+\eta\left(u_{\mathrm{f}}-u_{\mathrm{p}}\right)-1 \\
& \frac{\mathrm{d}^{2} u_{\mathrm{p}}}{\mathrm{d} y^{2}}=\frac{\gamma_{\mathrm{p}}}{\sqrt{D a_{\mathrm{p}}}} u_{\mathrm{p}}^{2}+\frac{u_{\mathrm{p}}}{D a_{\mathrm{p}}}+\eta\left(u_{\mathrm{p}}-u_{\mathrm{f}}\right)-1
\end{aligned}
$$

The pertinent no-slip boundary conditions at the walls of the channel read

$$
u_{\mathrm{f}}( \pm 1)=u_{\mathrm{p}}( \pm 1)=0
$$

Everywhere the original notation of Nield and Kuznetsov (2013) is used. Thus, $u_{\mathrm{f}}$ and $u_{\mathrm{p}}$ are the fluid velocities in the fracture phase (subscript $\mathrm{f}$ ) and the porous phase (subscript $\mathrm{p}$ ) of the bidisperse porous medium, respectively, $D a_{\mathrm{f}}$ and $D a_{\mathrm{p}}$ the corresponding Darcy numbers, $\gamma_{\mathrm{f}}$ and $\gamma_{\mathrm{p}}$ the Forchheimer parameters, $\eta$ is the coefficient for momentum transfer between the two phases and $y$ is the dimensionless transverse coordinate of the channel, $-1 \leq y \leq+1$. The term ' -1 ' on the right hand side of Eqs. (1) and (2) is the scaled form of the applied (constant) pressure gradient.

\section{Solution by Reduction to Normal Modes}

When the Forchheimer inertia terms are canceled $\left(\gamma_{\mathrm{f}}=\gamma_{\mathrm{p}}=0\right)$ the boundary value problem (1)-(3) becomes linear and can be solved by some well-established general methods. Here the powerful method of the normal modes known from the theory of small oscillations will be applied.

In the absence of the inertia terms Eqs. $(1,2)$ become

$$
\frac{\mathrm{d}^{2} u_{\mathrm{f}}}{\mathrm{d} y^{2}}=A u_{\mathrm{f}}-\eta u_{\mathrm{p}}-1, \quad \frac{\mathrm{d}^{2} u_{\mathrm{p}}}{\mathrm{d} y^{2}}=B u_{\mathrm{p}}-\eta u_{\mathrm{f}}-1,
$$

where the notation

$$
A=\eta+\frac{1}{D a_{\mathrm{f}}}, \quad B=\eta+\frac{1}{D a_{\mathrm{p}}}
$$

has been used. Substituting here

$$
u_{\mathrm{f}}(y)=a+U(y), \quad u_{\mathrm{p}}(y)=b+V(y)
$$

where

$$
a=\frac{\left(1+2 \eta D a_{\mathrm{p}}\right) D a_{\mathrm{f}}}{1+\eta\left(D a_{\mathrm{f}}+D a_{\mathrm{p}}\right)}, \quad b=\frac{\left(1+2 \eta D a_{\mathrm{f}}\right) D a_{\mathrm{p}}}{1+\eta\left(D a_{\mathrm{f}}+D a_{\mathrm{p}}\right)}
$$

Equation (4) reduce to

$$
\frac{\mathrm{d}^{2} U}{\mathrm{~d} y^{2}}=A U-\eta V, \quad \frac{\mathrm{d}^{2} V}{\mathrm{~d} y^{2}}=B V-\eta U,
$$


Although the constant terms of Eq. (4) have been eliminated by the substitution (6), the essential feature of Eq. (4), namely the momentum-coupling of the fracture and porous phase velocities $U$ and $V$ by the $\eta$ - terms are still present also in the transformed Eq. (8). Obviously, a direct elimination of one of the velocities can simply be accomplished, but this operation increases the order of the differential equation satisfied by the remaining velocity to 4 (see Nield and Kuznetsov 2005). The advantage of the method of reduction to normal modes which will be applied here consists of the fact that it allows for a decoupling of the system (8) without increasing the order of equations. The result of the reduction is a system of two decoupled normal oscillators. This method of the classical mechanics can be applied with success in many other areas of physics [for applications in heat transfer see Vadasz (2011) and Magyari (2007)].

The first step of the normal mode analysis is the transcription of Eq. (8) in the matrix form

$$
\left(\begin{array}{cc}
L-A & \eta \\
\eta & L-B
\end{array}\right)\left(\begin{array}{l}
U \\
V
\end{array}\right)=0
$$

where $L=\mathrm{d}^{2} / \mathrm{d} y^{2}$. Then one performs the matrix-similarity transformation

$$
S^{-1}\left(\begin{array}{cc}
L-A & \eta \\
\eta & L-B
\end{array}\right) S S^{-1}\left(\begin{array}{l}
U \\
V
\end{array}\right)=0
$$

where $S$ is some non-singular $2 \times 2$ matrix with the constant matrix elements $S_{i j}$ and $S^{-1}$ stands for the inverse matrix of $S$. Furthermore we denote

$$
S^{-1}\left(\begin{array}{l}
U \\
V
\end{array}\right)=\left(\begin{array}{l}
Z_{1} \\
Z_{2}
\end{array}\right) \text { which yields }\left(\begin{array}{l}
U \\
V
\end{array}\right)=S\left(\begin{array}{l}
Z_{1} \\
Z_{2}
\end{array}\right)
$$

Then we chose $S$ such that in Eq. (10) the transformed matrix $S^{-1}$ (..) $S$ becomes diagonal. This requirement is satisfied by the choice

$$
S=\left(\begin{array}{cc}
-c & 1 \\
1 & c
\end{array}\right), \quad S^{-1}=\frac{1}{1+c^{2}}\left(\begin{array}{cc}
-c & 1 \\
1 & c
\end{array}\right)
$$

where

$$
c=\frac{A-B}{2 \eta}+\sqrt{1+\left(\frac{A-B}{2 \eta}\right)^{2}}
$$

Equation (10) diagonalized in this way results in

$$
\left(\begin{array}{cc}
L-\omega_{1}^{2} & 0 \\
0 & L-\omega_{2}^{2}
\end{array}\right)\left(\begin{array}{l}
Z_{1} \\
Z_{2}
\end{array}\right)=0
$$

where

$$
\omega_{1,2}^{2}=\eta\left(\frac{A+B}{2 \eta} \pm \sqrt{1+\left(\frac{A-B}{2 \eta}\right)^{2}}\right)
$$

The matrix Eq. (14) yields for the normal modes $Z_{1}$ and $Z_{2}$ the two decoupled differential equations

$$
\frac{\mathrm{d}^{2} Z_{1,2}}{\mathrm{~d} y^{2}}=\omega_{1,2}^{2} Z_{1,2}
$$


which admit the general solutions

$$
Z_{1,2}(y)=C_{1,2} \cosh \left(\omega_{1,2} y+\alpha_{1,2}\right)
$$

The governing Eq. (4) and the boundary conditions (3) are invariant under the reflection $y \rightarrow-y$ of the transverse coordinate. Therefore, the solutions which possess this symmetry property must be even functions of $y$. This requires in turn that the integration constants $\alpha_{1,2}$ must be zero. Accordingly, Eqs. (6) and (17) along with the second matrix Eq. (11) lead to the following general form of the solutions

$$
\begin{aligned}
& u_{\mathrm{f}}(y)=a-c Z_{1}+Z_{2}=a-c C_{1} \cosh \left(\omega_{1} y\right)+C_{2} \cosh \left(\omega_{2} y\right) \\
& u_{\mathrm{p}}(y)=b+Z_{1}+c Z_{2}=b+C_{1} \cosh \left(\omega_{1} y\right)+c C_{2} \cosh \left(\omega_{2} y\right)
\end{aligned}
$$

Now the remaining two integration constants $C_{1,2}$ can easily be determined from the boundary conditions (3). Thus the exact solution of our boundary value problem reads

$$
\begin{aligned}
& u_{\mathrm{f}}(y)=a-\frac{c(a c-b)}{1+c^{2}} \frac{\cosh \left(\omega_{1} y\right)}{\cos h\left(\omega_{1}\right)}-\frac{a+b c}{1+c^{2}} \frac{\cos h\left(\omega_{2} y\right)}{\cos h\left(\omega_{2}\right)} \\
& u_{\mathrm{p}}(y)=b-\frac{c(a+b c)}{1+c^{2}} \frac{\cosh \left(\omega_{2} y\right)}{\cos h\left(\omega_{2}\right)}+\frac{a c-b}{1+c^{2}} \frac{\cos h\left(\omega_{1} y\right)}{\cos h\left(\omega_{1}\right)}
\end{aligned}
$$

The above Eq. (19) is equivalent to Eq. (2.16) of Nield and Kuznetsov (2005) which is the solution of a boundary value problem of fourth order obtained by a direct elimination of $u_{\mathrm{p}}$ from the system of Eq. (4).

\section{Discussion}

\subsection{General Features of the Exact Solutions}

Equations (19) and (20) show clearly that the velocity fields $u_{\mathrm{f}}(y)$ and $u_{\mathrm{p}}(y)$ are symmetric with respect to the midplane $y=0$ of the channel. It is also easy to see that $u_{\mathrm{f}}(y)$ and $u_{\mathrm{p}}(y)$ satisfy all the no-slip conditions (3). Furthermore it can be shown that the largest fluid velocities are always obtained for $y=0, u_{\mathrm{f}, \max }=u_{\mathrm{f}}(0), u_{\mathrm{p}, \max }=u_{\mathrm{p}}(0)$. The velocity gradients at the walls are given by the relationships

$$
\begin{aligned}
& u_{\mathrm{f}}^{\prime}(-1)=-u_{\mathrm{f}}^{\prime}(+1)=\frac{c(a c-b)}{1+c^{2}} \omega_{1} \tan h\left(\omega_{1}\right)+\frac{a+b c}{1+c^{2}} \omega_{2} \tan h\left(\omega_{2}\right), \\
& u_{\mathrm{p}}^{\prime}(-1)=-u_{\mathrm{p}}^{\prime}(+1)=\frac{c(b c+a)}{1+c^{2}} \omega_{2} \tan h\left(\omega_{2}\right)-\frac{a c-b}{1+c^{2}} \omega_{1} \tan h\left(\omega_{1}\right)
\end{aligned}
$$

As an illustration of the above features, in Figs. 1 and 2 the velocity profiles $u_{\mathrm{f}}(y)$ and $u_{\mathrm{p}}(y)$ have been plotted for the parameter values $\left(\eta=1, D a_{\mathrm{f}}=0.1, D a_{\mathrm{p}}=0.01\right)$ and $(\eta=1$ and $\left.D a_{\mathrm{f}}=0.01, D a_{\mathrm{p}}=0.001\right)$, respectively. This choice is motivated by the values selected by Nield and Kuznetsov (2013) as being 'representative' for the Forchheimer-extension of the present model. It is seen that all the velocity profiles become flatter as the Darcy numbers decrease. Therefore, this property, which enhances the stability of the shear flows (Nield and Kuznetsov 2013), is present already at the linear version of the model (which neglects the Forchheimer inertia effects). 
Fig. 1 Velocity profiles in the fracture and porous phase for the indicated values of the parameters. The maximum velocities are $u_{\mathrm{f}}(0)=$ $0.085215, u_{\mathrm{p}}(0)=0.010736$

Fig. 2 Velocity profiles in the fracture and porous phase for the indicated values of the parameters. The maximum velocities are $u_{\mathrm{f}}(0)=$ $0.009910, u_{\mathrm{p}}(0)=0.001009$
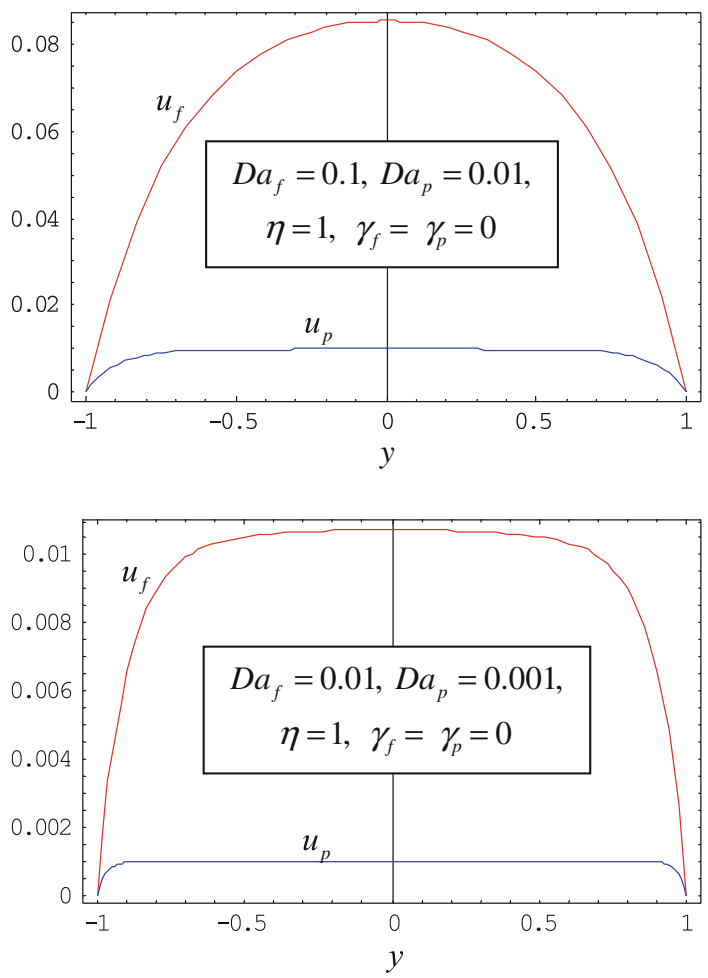

\subsection{The Weak Coupling Limit $\eta \rightarrow 0$}

The mathematical complexity of the boundary value problem (1)-(3) originates actually from the momentum transfer coefficient $\eta$ which couples the fluid flows in the fracture and porous phases to each other. When this coupling can be neglected, the system $(1,2)$ decays in two independent equations of the same form which describe the forced convection channel flow in a usual ("monodisperse") Darcy-Brinkman-Forchheimer porous medium. The solution of this type of (nonlinear) flow problem has been given in a comprehensive paper of by Nield et al. (1996) in terms of elliptic integrals. In the case of the linear Darcy-Brinkman model $\left(\gamma_{\mathrm{f}}=\gamma_{\mathrm{p}}=0\right)$ the weak-coupling limit of the negligible momentum transfer between the flow phases $(\eta=0)$ can easily be obtained from the exact solutions (19) and (20). Indeed, bearing in mind that for $\eta=0, a=D a_{\mathrm{f}}, b=D a_{\mathrm{p}}, \omega_{1}=D a_{\mathrm{f}}^{-1 / 2}, \omega_{2}=D a_{\mathrm{p}}^{-1 / 2}$ and $c=\infty$, one obtains the simple result

$$
u_{\mathrm{f}}(y)=D a_{\mathrm{f}}\left[1-\frac{\cosh \left(D a_{\mathrm{f}}^{-1 / 2} y\right)}{\cosh \left(D a_{\mathrm{f}}^{-1 / 2}\right)}\right], \quad u_{\mathrm{p}}(y)=D a_{\mathrm{p}}\left[1-\frac{\cosh \left(D a_{\mathrm{p}}^{-1 / 2} y\right)}{\cosh \left(D a_{\mathrm{p}}^{-1 / 2}\right)}\right]
$$

\subsection{The Strong Coupling $\eta \rightarrow \infty$}

In the strong coupling limit $(\eta \rightarrow \infty)$ it is expected that, as an effect of the enhanced momentum transfer between the phases, the velocity profiles $u_{\mathrm{f}}(y)$ and $u_{\mathrm{p}}(y)$ approach each other. This is actually the case. As $\eta \rightarrow \infty$, to the order $\eta^{-1}$ the velocities (19), (20) 
become

$$
\begin{aligned}
u_{\mathrm{f}}(y)= & \left(a_{0}+\frac{a_{1}}{\eta}\right)\left[1-\frac{\cos h\left(a_{0}^{-1 / 2} y\right)}{\cos h\left(a_{0}^{-1 / 2}\right)}\right] \\
& +\frac{a_{0}^{3 / 2}}{16 \eta}\left(\frac{1}{D_{\mathrm{f}}}-\frac{1}{D_{\mathrm{p}}}\right)^{2}\left[y \tan h\left(a_{0}^{-1 / 2} y\right)-\tan h\left(a_{0}^{-1 / 2} y\right)\right] \frac{\cos h\left(a_{0}^{-1 / 2} y\right)}{\cos h\left(a_{0}^{-1 / 2}\right)}, \\
u_{\mathrm{p}}(y)= & \left(b_{0}+\frac{b_{1}}{\eta}\right)\left[1-\frac{\cos h\left(b_{0}^{-1 / 2} y\right)}{\left.\cos h\left(b_{0}^{-1 / 2}\right)\right]}\right. \\
& +\frac{b_{0}^{3 / 2}}{16 \eta}\left(\frac{1}{D_{\mathrm{f}}}-\frac{1}{D_{\mathrm{p}}}\right)^{2}\left[y \tan h\left(b_{0}^{-1 / 2} y\right)-\tan h\left(b_{0}^{-1 / 2} y\right)\right] \frac{\cos h\left(b_{0}^{-1 / 2} y\right)}{\cos h\left(b_{0}^{-1 / 2}\right)}
\end{aligned}
$$

where

$$
a_{0}=b_{0}=\frac{2 D a_{\mathrm{f}} D a_{\mathrm{p}}}{D a_{\mathrm{f}}+D a_{\mathrm{p}}}, \quad a_{1}=\frac{D a_{\mathrm{f}}\left(D a_{\mathrm{f}}-D a_{\mathrm{p}}\right)}{\left(D a_{\mathrm{f}}+D a_{\mathrm{p}}\right)^{2}}, \quad b_{1}=\frac{D a_{\mathrm{p}}\left(D a_{\mathrm{p}}-D a_{\mathrm{f}}\right)}{\left(D a_{\mathrm{f}}+D a_{\mathrm{p}}\right)^{2}}
$$

Now it is clearly seen that, as $\eta \rightarrow \infty$, in the leading order approximation the velocities (24) and (25) become coincident,

$$
u_{\mathrm{f}}(y)=u_{\mathrm{p}}(y)=a_{0}\left[1-\frac{\cosh \left(a_{0}^{-1 / 2} y\right)}{\cosh \left(a_{0}^{-1 / 2}\right)}\right]
$$

In this respect it is instructive to compare the limiting solutions (26) to the exact ones given by Eqs. (19) and (20). Doing this for the values of $D a_{\mathrm{f}}$ and $D a_{\mathrm{p}}$ used in Fig. $1 \mathrm{a}, \mathrm{b}$, there turns out that (at the scales of these Figures) the limiting profiles (26) and the exact ones become undistinguishable at $\eta=10^{4}$ and $\eta=10^{5}$ for the Darcy numbers selected in the Figs. 1 and 2, respectively.

\subsection{The Effect of Forchheimer Drag}

In order to get a first sense for the effect of Forchheimer inertia, in Fig. 3a, b the numerical solution $\left(u_{\mathrm{f}}, u_{\mathrm{p}}\right)$ of the nonlinear problem (1)-(3) represented by the black curves and corresponding to the parameter values $\left(\gamma_{\mathrm{f}}=\gamma_{\mathrm{p}}=\eta=1, D a_{\mathrm{f}}=0.1, D a_{\mathrm{p}}=0.01\right)$ has been compared to the exact solution (19), (20) of the linear problem $(3,4)$. The latter solutions coincide, respectively, with the red and blue curves of Fig. 1, associated with the parameter values $\left(\gamma_{\mathrm{f}}=\gamma_{\mathrm{p}}=0, \eta=1, D a_{\mathrm{f}}=0.1, D a_{\mathrm{p}}=0.01\right)$. At the same time, in Fig. 3a,b the above-mentioned solutions can also be compared to the zero-coupling solutions (green curves) given by Eq. (22) and associated with the parameter values $\left(\gamma_{\mathrm{f}}=\gamma_{\mathrm{p}}=\eta=0, D a_{\mathrm{f}}=0.1, D a_{\mathrm{p}}=0.01\right)$.

The inspection of Fig. 3a, b leads to the following conclusions.

1. The black and red curves of Fig. 3a are nearly overlapping which shows that the effect of the Forchheimer drag on the fracture phase velocity $u_{\mathrm{f}}$ is negligibly small. The 
Fig. 3 a Velocity profiles $u_{\mathrm{f}}$ in the fracture phase for the indicated values of the Darcy numbers and the values $\gamma_{\mathrm{f}}=\gamma_{\mathrm{p}}=\eta=1$ (black curve, nonlinear problem),

$\gamma_{\mathrm{f}}=\gamma_{\mathrm{p}}=0, \eta=1$ (red curve, linear problem, Eq. 19) and $\gamma_{\mathrm{f}}=\gamma_{\mathrm{p}}=\eta=0$ (green curve, linear problem, with zero-coupling, first Eq. 22), respectively. The maximum flow velocities are $u_{\mathrm{f}}(0)=0.091549$ (green), $u_{\mathrm{f}}(0)=0.085215$ (red), $u_{\mathrm{f}}(0)=0.083546$ (black).

b Velocity profiles $u_{\mathrm{p}}$ in the porous phase for the indicated values of the Darcy numbers and the values $\gamma_{\mathrm{f}}=\gamma_{\mathrm{p}}=\eta=1$ (black curve, nonlinear problem), $\gamma_{\mathrm{f}}=\gamma_{\mathrm{p}}=0, \eta=1$ (blue curve, linear problem, Eq. 20) and $\gamma_{\mathrm{f}}=\gamma_{\mathrm{p}}=\eta=0$ (green curve, linear problem, with zero-coupling, second Eq. 22), respectively. The black and blue curves are highly overlapping. The maximum flow velocities are $u_{\mathrm{p}}(0)=0.010736$ (blue), $u_{\mathrm{p}}(0)=0.010708$ (black), $u_{\mathrm{p}}(0)=0.009999$ (green)
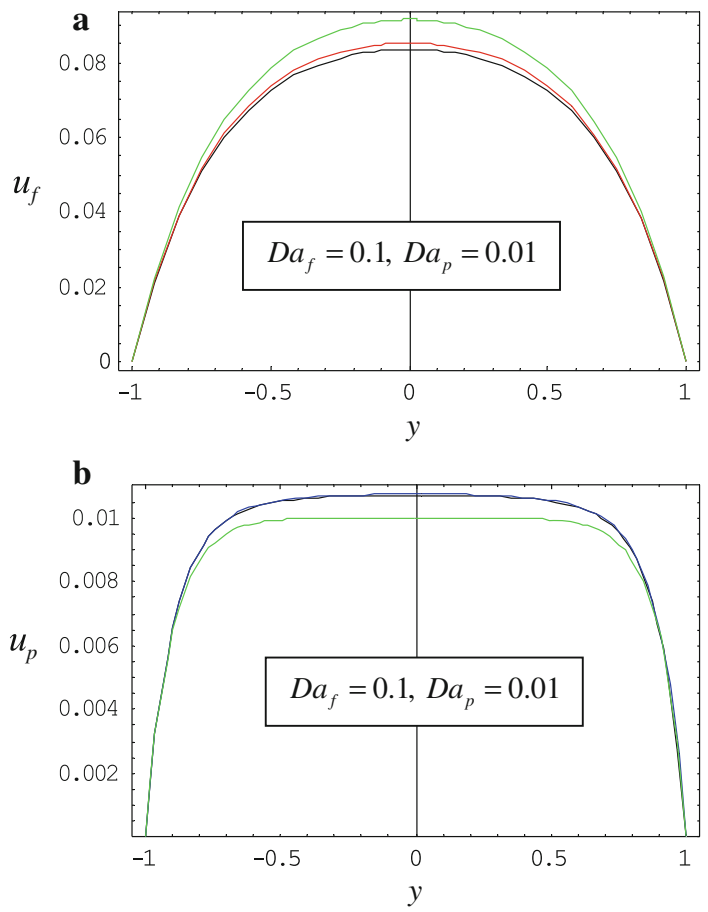

Forchheimer inertia (black curve) reduces the maximum velocity of the red curve by approximately $2 \%$. Even the zero-coupling limit $(\eta=0)$ of the linear model (green curve) yields a reasonable approximation to the nonlinear result (black curve).

2. For the porous phase velocities $u_{\mathrm{p}}$ plotted in Fig. 3b, all the above effects become even enhanced. Now the blue and black curves are widely overlapping. The Forchheimer inertia (black curve) reduces the maximum velocity of the blue curve by only $0.3 \%$. For the parameter values selected in Fig. 2 (Darcy numbers diminished by one order of magnitude) all the mentioned effects become enhanced once more.

3. The degree of flatness of the porous phase velocities $u_{\mathrm{p}}$ is larger than that of the fracture phase velocities $u_{\mathrm{f}}$. This feature becomes manifested at all the three levels of approximation (linear model with $\eta=0$, linear model with $\eta=1$ and nonlinear model with $\eta=1)$.

\section{Summary and Conclusions}

The linear Darcy-Brinkman model of the high speed flow in a bidisperse porous medium proposed by Nield and Kuznetsov (2005) as well as its nonlinear counterpart which includes the Forchheimer drag (Nield and Kuznetsov 2013) has been revisited in this paper. For the steady unidirectional flow in a parallel plane channel the exact analytical solution of the linear model has been worked out by the normal-mode analysis of the governing equations. This 'order-conserving' method which reduces the problem to that of a decoupled system of normal oscillators is a general and powerful alternative to the elimination method which necessarily 
increases the order of the differential equations satisfied by the individual variables (in the present case from 2 to 4 ).

In order to evaluate the nonlinear effect of the Forchheimer inertia, the exact solution of the linear model has been compared to the numerical solution of the nonlinear model for some representative values of the parameters. The main result of this analysis is that the contribution of the Forchheimer drag to the linear model is negligibly small. Surprisingly, even the zero-coupling limit $(\eta=0)$ of the fracture and porous flow phases yields an acceptable approximation to the solution of the nonlinear model. In this respect an extended parameter study requires further research effort.

\section{References}

Magyari, E.: Normal mode analysis of the fully developed free convection flow in a vertical slot with open to capped ends. Heat Mass Transfer 43, 827-832 (2007)

Nield, D.A., Bejan, A.: Convection in Porous Media. Springer, Berlin (2006)

Nield, D.A., Junqueira, S.L.M., Lage, J.L.: Forced convection in a fluid-saturated porous-medium channel with isothermal or isoflux boundaries. J. Fluid Mech. 322, 201-214 (1996)

Nield, D.A., Kuznetsov, A.V.: Heat transfer in bidisperse porous media. In: Ingham, D.B., Pop, I. (eds.) Transport Phenomena in Porous Media III, pp. 34-59. Elsevier, Oxford (2005)

Nield, D.A., Kuznetsov, A.V.: A note on modeling high speed flow in a bidisperse porous medium. Transp. Porous Media 96, 495-499 (2013)

Vadasz, P.: Basic natural convection in a vertical porous layer differentially heated from its sidewalls subject to lack of local thermal equilibrium. Int. J. Heat Mass Transfer 54, 2387-2396 (2011) 